\title{
Accurate myocardial blood flow measurements: Quality from start to finish is key to success
}

\author{
James Case ${ }^{\mathrm{a}}$ \\ a Cardiovascular Imaging Technologies, Kansas City, MO
}

Received Aug 29, 2016; accepted Aug 29, 2016

doi: $10.1007 / \mathrm{s} 12350-016-0690-1$

\begin{abstract}
Overall repeatability and accuracy of myocardial blood flow measurements depend on the quality of the image data, the proper use of the quantitative software, and the training of clinical staff. Unlike most imaging tests, absolute blood flow measurements have the potential to provide accurate information beyond what is seen visually. This additive information has been shown to help identify multivessel disease, balanced ischemia, true normal studies, and quality control problems that may not be visible in the relative perfusion pattern. Absolute blood flow and flow reserve measurements have been shown to be useful and reproducible on a wide array of imaging systems, radiotracer delivery systems, and software approaches. Regardless of the technology or technique, these benefits can only be achieved when the quality of the study is sufficient and the clinical staff is properly trained on its implementation.
\end{abstract}

\section{INTRODUCTION}

As with anything in life, a commitment to quality is the key to success. This is especially true when it comes to the quantitative assessment of myocardial blood flow (MBF) and myocardial flow reserve (MFR) in cardiac PET. All the PET software and hardware systems used today for MFR assessment have their strengths and weaknesses. Most are cleared by the United States Food and Drug Administration and have numerous publications demonstrating their efficacy. However, it is the responsibility of every user to understand their software, understand how to assess its quality, and, importantly, when to believe or not believe its results.

In the manuscript entitled, "Consistent tracer administration profile improves test-retest repeatability

See related article, pp. 929-941

\footnotetext{
Reprint requests: James Case, Cardiovascular Imaging Technologies, Kansas City, MO; jcase@cvit.com

J Nucl Cardiol 2018;25:942-6.

$1071-3581 / \$ 34.00$

Copyright (c) 2018 American Society of Nuclear Cardiology.

of myocardial blood flow quantification with ${ }^{82} \mathrm{Rb}$ dynamic PET imaging," Klein et.al. examined the repeatability of MBF measurements when a constant activity rate of administration of ${ }^{82} \mathrm{Rb}$ is used. Their conclusion was that constant activity (CA) administration of ${ }^{82} \mathrm{Rb}$ led to more repeatable MBF estimates when compared to current constant flow (CF) administration. In particular, the authors presented data demonstrating that an improvement in the repeatability of MBF and MFR was most significant when a simplified retention model (SRM) is used for modeling the tracer uptake (test-retest repeatability coefficient of $23 \%$ (CA) vs $49 \%$ $(\mathrm{CF})$ ). This effect was less important when a one-tissue compartment model (1TCM) model was used (test-retest repeatability coefficient of $20 \%$ (CA) vs $27 \%$ (CF)), implying a potentially greater robustness when the $\mathrm{CF}$ infusion was used.

The overall myocardial blood flow and flow reserve repeatability coefficients for the CF system using the SRM would seem high when compared to studies with larger patient groups. Yoshida et. al. considered 135 subjects imaged using a $\mathrm{CF},{ }^{82} \mathrm{Rb}$ generator and observed the high degree of correlation of myocardial blood flow values determined with a SRM vs ${ }^{13} \mathrm{~N}$ ammonia using compartmental modeling $(r=0.98) .{ }^{1}$ In addition, Murthy et.al. examined the variability of myocardial reserve for a 1TCM and a SRM in 2,783 consecutive patients undergoing a clinically indicated rest-stress ${ }^{82} \mathrm{Rb}$ study, and concluded that the model for 
measuring arterial input function was more important than the kinetic model in determining reproducibility. In particular, when the method for determining the arterial input function was the same, there was a strong correlation between the various extraction models, $(r>0.90) .^{2}$ In a separate comparison of ten different ${ }^{82} \mathrm{Rb}$ flow quantitation programs and a constant activity infusion system, a high degree of correlation was observed between absolute flow and reserve values, as long as the same extraction model and input function model were used. ${ }^{3}$

Given the small number of patients in each cohort, the current study from Klein et.al. ( $n=10$ in the constant flow cohort), maintaining the quality of the data is essential for obtaining meaningful results. The authors examined several quality factors that could influence their data such as the ROI placement and system dead times. They also examined one particular subject that had resting blood flow values that would be considered non-physical ( $>2.0 \mathrm{ml} / \mathrm{gm} / \mathrm{min})$. The authors identified several possible quality explanations for this subject, and suggested there may have been a problem with the injection site and clearance of activity from the blood pool.

Obtaining good quality, reliable myocardial blood flow measurements that confer a benefit to patient management is more than purchasing a software program and plugging it into the department. Clinicians must have a complete understanding of the principles of measuring flow, and use appropriate imaging protocols that are compatible with the software used. In addition, clinical staff must be well trained to ensure the accuracy and reproducibility of blood flow measurements in clinical practice.

\section{OPTIMIZING THE PROTOCOL}

There is a wide variation of cardiac PET systems in use in the United States and worldwide. In the US, roughly half of the PET systems in use utilize dedicated, line source systems for attenuation correction, and the other half use hybrid list-mode PET/CT systems. Both types of systems have been validated for performing the dynamic study necessary for calculating absolute blood flow, despite significant differences in protocols used by these systems (see Figures 1, 2).

PET systems can acquire data in either a framemode or a list-mode. List-mode acquisitions record each individual coincidence event as it is received by the scanner. Following the acquisition, the user can rebin those events into an arbitrary number of gated, static, and dynamic studies. List mode can be very useful for blood flow studies because there is no need to acquire

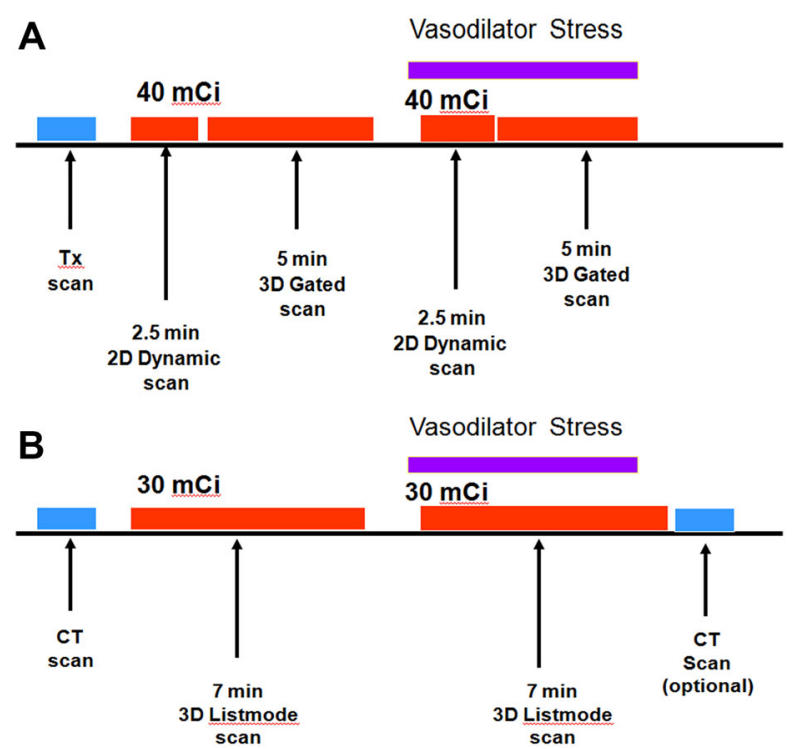

Figure 1. Sample imaging protocol for acquiring perfusion, gating, and dynamic PET data using a frame-mode system (a) and a list-mode system (b).

separate acquisitions for the dynamic and gated studies. This can potentially reduce radiation dose and improve laboratory efficiency. To accomplish this, these systems must be capable of accurately acquiring quantitative PET data across a wide range of count rates. When highsensitivity, low dead-time list-mode scanners are available, infused activity can be $30 \mathrm{mCi}(1,110 \mathrm{MBq})$ of ${ }^{82} \mathrm{Rb}$ or lower (Table 1$){ }^{3}$

Many of the systems in use today cannot be acquired at the high count rates necessary to perform the dynamic study in a 3D septa-retracted mode because of limitations on dead time. For these systems, it is necessary to acquire the dynamic study in the lower sensitivity 2D septa-extended mode. ${ }^{4}$ For those systems with retractable septa, this problem can be overcome by acquiring the dynamic, high count data in 2D and followed by a 3D perfusion study, thereby taking advantage of the radioactive decay of the ${ }^{82} \mathrm{Rb}$. With this type of study, there is no need to employ a list-mode acquisition. ${ }^{5}$ This protocol can acquire the necessary data; however, staff must be trained on the rapid transition needed to switch from the dynamic to gated scan.

\section{PROCESSING}

The reconstruction of quantitative dynamic myocardial perfusion PET studies should always be performed using an iterative reconstruction algorithm, including all necessary corrections: attenuation, scatter, prompt 

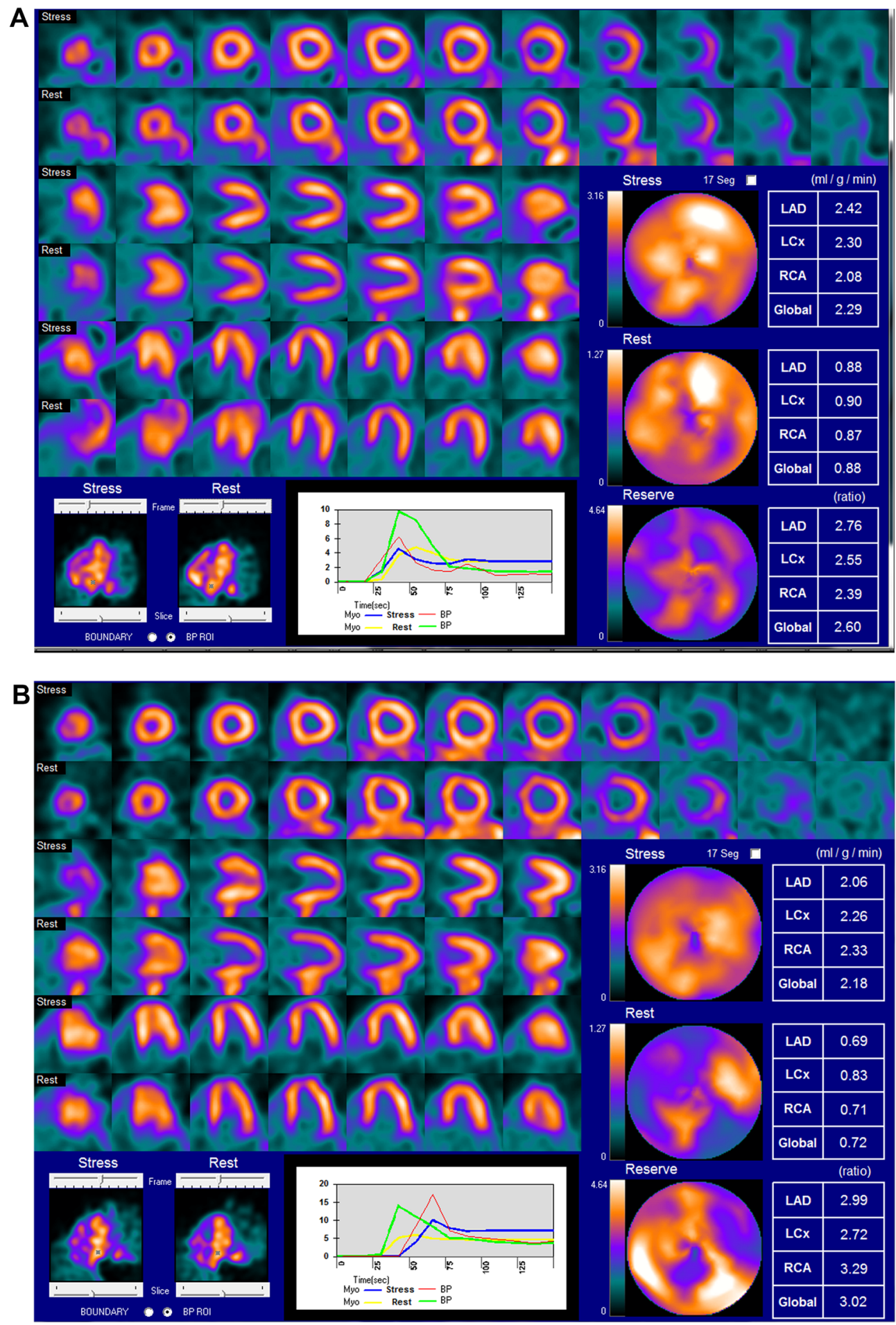

Figure 2. Example of two normal cardiac PET perfusion and flow studies (different patients) acquired using a list-most system (a) and a frame-mode system (b).

gamma, randoms, dead time, and radioactive decay. Failure to include any of these corrections will introduce quantitative uncertainty and bias in the flow results. ${ }^{6}$
The flow processing must be performed according to the techniques specific to the processing software used. Quality control rules that are applicable to one 
Table 1. Summary of the advantages and disadvantages of various PET acquisition strategies for measuring MBF and MFR

\begin{tabular}{|c|c|c|}
\hline & Advantage & Limitation \\
\hline $\begin{array}{l}\text { List-mode dynamic } \\
\text { study }\end{array}$ & $\begin{array}{l}\text { Single acquisition for static, gated, and } \\
\text { dynamic studies. Improves laboratory } \\
\text { efficiency and potentially lowering } \\
\text { patient dosage }\end{array}$ & $\begin{array}{l}\text { System must be able to accurately acquire } \\
\text { at high and low count rates. Can be } \\
\text { challenging in 3D }\end{array}$ \\
\hline $\begin{array}{l}\text { Frame-mode } \\
\text { dynamic study }\end{array}$ & $\begin{array}{l}\text { Uses different dynamic and gated } \\
\text { acquisition parameters, 2D for } \\
\text { dynamic and 3D for gated. Less dead } \\
\text { time }\end{array}$ & $\begin{array}{l}\text { Longer acquisition times and potentially } \\
\text { high injected dosages depending on } \\
\text { protocol }\end{array}$ \\
\hline 2D imaging mode & $\begin{array}{l}\text { Low dead time for dynamic and gated } \\
\text { studies. No prompt gamma } \\
\text { correction. Less scatter. Accurate } \\
\text { quantitative values }\end{array}$ & $\begin{array}{l}\text { Requires higher injected } \\
\text { radiopharmaceutical dosages }\end{array}$ \\
\hline 3D imaging mode & $\begin{array}{l}\text { Can reduce patient radiation dose if } \\
\text { required }\end{array}$ & $\begin{array}{l}\text { Requires PET system capable of accurately } \\
\text { recording counts at very high count } \\
\text { rates during the dynamic while having } \\
\text { good sensitivity during the gated study }\end{array}$ \\
\hline
\end{tabular}

software program may not be appropriate or accurate on a different software package. For example, 1TCM applications require the blood pool ROI be placed within the left ventricle. ${ }^{7}$ In contrast, most SRM applications require the blood pool ROI be placed in the left atrium. ${ }^{1,3}$ The location of these ROIs is crucial in obtaining accurate blood flow measurement, and technologists must be trained on appropriate processing techniques. $^{8}$

\section{INTERPRETATION}

Combining absolute blood flow and flow reserve into the interpretation of myocardial perfusion PET images requires an understanding of the imaging process: acquisition, quality control, and processing. Independent of flow model (SRM or 1TCM) or infusion (CA or CF), absolute myocardial blood flow analysis has been demonstrated to provide valuable patient management information, beyond what can be seen visually. ${ }^{9}$

One of the most important goals of using absolute blood flow is the assessment of multivessel disease and balanced ischemia. In a study of 120 patients it was demonstrated that myocardial blood flow reserve was an independent predictor of three vessel disease $(\mathrm{p}=0.015)$. $\left(\mathrm{CA}-1 \mathrm{TCM},{ }^{10}\right)$. In addition, preserved coronary flow reserve effectively rules out high risk coronary disease when MFR $>1.93$ with a negative predictive value of $93 \%\left(\mathrm{CF}-1 \mathrm{TCM},{ }^{11}\right)$. The persistence of the vasodilatory effect has also been investigated using MBF measurements. For one of the most commonly used vasodilator, regadenoson, MFR has been shown to be highly reproducible when using a 1-minute delay for starting the ${ }^{82} \mathrm{Rb}$ infusion post regadenoson injection: $\operatorname{MFR}(60 \mathrm{~s})=2.79 \pm 0.56$ or $20 \%\left(\mathrm{CF}-\mathrm{SRM},{ }^{12}\right)$.

Trusting these numbers begins with applying proper quality review of every image. Clinicians must get appropriate training on how these images are created, processed, and how to identify when quality control problem have occurred. The importance of understanding how quality issues can influence the repeatability of myocardial blood flow measurements is essential to obtaining reliable and useful data, independent of the generator or software used.

\section{CONCLUSION}

Klein et.al. have presented some of the important challenges in performing MBF and MFR assessments and when robust, quality data are available, these values can be highly reproducible. The widespread availability of absolute blood flow and reserve measurements may revolutionize nuclear cardiology. However, if these measurements are not done accurately, these quantities will be of little value to the practicing clinician. When integrating myocardial blood flow measurements into practice, it is vitally important that all of the clinical staff be trained on the entire protocol, including patient preparation, vasodilator stressing, imaging parameters, quality control, processing, and interpretation. 
Absolute myocardial blood flow measurements can add tremendous value to the interpretation. It can be implemented in a variety of ways on a diverse set of instrumentation using numerous software approaches. However, regardless of the software, generator, or instrumentation used, the accuracy and value of the absolute blood flow measurements will rely on the commitment of the laboratory staff to quality.

\section{References}

1. Yoshida K, Mullani N, Gould KL. Coronary flow and flow reserve by PET simplified for clinical applications using rubidium- 82 or nitrogen-13-ammonia. J Nucl Med. 1996;37(10):1701-12.

2. Murthy VL, Lee BC, Sitek A, Naya M, Moody J, Polavarapu V, Ficaro EP, Di Carli MF. Comparison and prognostic validation of multiple methods of quantification of myocardial blood flow with 82Rb PET. J Nucl Med. 2014;55(12):1952-8.

3. Nesterov SV, Deshayes E, Sciagrà R, Settimo L, Declerck JM, Pan X-B, Yoshinaga K, Katoh C, Slomka PJ, Germano G, Han C, Aalto V, Alessio AM, Ficaro EP, Lee BC, Nekolla SG, Gwet KL, deKemp RA, Klein R, Dickson J, Case JA, Timothy Bateman MD, Prior JO, Knuuti JM (2014) Quantification of myocardial blood flow in absolute terms using 82Rb PET imaging: the RUBY-10 study. J Am Coll Cardiol Img 7(11):1119-1127

4. Tout D, Tonge C, Muthu S, Arumugam P. Assessment of a protocol for routine simultaneous myocardial blood flow measurement and standard myocardial perfusion imaging with rubidium- 82 on a high count rate positron emission tomography system. Nucl Med Commun. 2012;33:1202-11.
5. Case JA, Van Vickle S, Courter SA, Burgett EV, Bateman TM (2014) A rapid protocol for measuring perfusion, gating and absolute blood flow using dedicated frame-mode 3D PET scanner. J Nucl Cardiol 21:768 (abstract)

6. Moody JB, Lee BC, Crobett JR, Ficaro EP, Murthy VL. Precision and accuracy of clinical quantification of myocardial blood flow by dynamic PET: a technical perspective. J Nucl Cardiol. 2015;22:935-51.

7. Efseaff M, Klein R, Ziadi MC, Beanlands RS, deKemp RA (2012) Short-term repeatability of resting myocardial blood flow measurements using rubidium-82 PET imaging. J Nucl Cardiol 19:997-1006

8. Case JA, Bateman TM. Taking the perfect nuclear image: quality control, acquisition, and processing techniques for cardiac SPECT, PET, and hybrid imaging. J Nucl Cardiol. 2013;20(5):891-907.

9. Bateman TM, Gould KL, Di Carli MF (2015) Proceedings of the cardiac PET summit, 12 May 2014, Baltimore, MD 3: quantitation of myocardial blood flow. J Nucl Cardiol 22:571-578

10. Ziadi MC, deKemp RA, Williams K, Guo A, Renauld KM, Chow BJW, Klein R, Ruddy TD, Aung M, Garrard L, Beanlands RSB (2012) Does quantification of myocardial flow reserve using rubidium-82 positron emission tomography facilitate detection of multivessel coronary artery disease? J Nucl Cardiol 19: 670-680

11. Naya M, Murthy VL, Taqueti VR, Foster CR, Klein J, Garber M, Dorbala S, Hainer J, Blankstein R, Resnic F, Di Carli MF (2014) Preserved coronary flow reserve effectively excludes high-risk coronary artery disease on angiography. J Nucl Med 55:248-255

12. Bateman TM, Case JA, Courter SA, Jensen J, Burgett EV, Van Vickle S (2016) Time dependence of myocardial blood flow reserve Measurements following regadenoson Rubidium-82 Myocardial Perfsuion PET: new data supporting longer infusion delay. J Nucl Cardio 23(4):309-02 (abstract) 